\title{
The theory of direct magnetoelectric effect in the bilayer system of ferromagnetic-piezoelectric
}

\author{
V. N. Nechaev and A. V. Shuba* \\ Department of Mathematics and Physical - Mathematical Modelling \\ Voronezh State Technical University, Moskowsky Prospect Street \\ Voronezh 394026, Russian Federation \\ *shandvit@rambler.ru
}

Received 16 December 2015; Accepted 4 February 2016; Published 28 March 2016

\begin{abstract}
The theory of direct magnetoelectric (ME) effect in a bilayer of ferromagnetic and piezoelectric is developed taking into account nonuniform distribution of electrical field on a piezoelectric layer thickness. The simultaneous solution of the motion equations for piezoelectric and ferromagnetic mediums allowed to numerically and analytically calculate the dependence of natural mechanical oscillation on structure parameters and to determine the dependence of ME effect coefficient on frequency of the variable magnetic field.

Keywords: Bilayer system; magnetoelectric effect; natural frequency of oscillations; longitudinal magnetoelectric voltage coefficient; nonuniform electric field.
\end{abstract}

\section{Introduction}

The natural multiferroics have the magnetoelectric (ME) effect that is the magnetic field is transformed into electric one (direct ME effect) or, on contrary, the electric field is transformed into magnetic one (reverse ME effect). Both the effects are used in practice, for example, as the high sensitive transducers of magnetic fields, the electrically controlled sources of variable magnetic field, the new types of magnetic memory and logical elements. ${ }^{1-3}$ However, the values of ME interaction coefficients, $\alpha_{E}, \alpha_{H}$ in it are negligible and observed at low temperature and high external field. ${ }^{4}$

The essential increase of the ME effect is archived in the artificially created layered composites of piezoelectric and ferromagnetic. ${ }^{5-7}$ In this case, the essential increase of ME effect is provided by the possibility of using the components with high magnitudes of magnetostriction and piezomodule, the application of external fields whose frequencies are close to the frequency of natural acoustic oscillation of system. In this connection, the many experimental researches ${ }^{6,8,9}$ as well as theoretical ones ${ }^{10-12}$ are devoted to multi-layered composites of piezoelectric and ferromagnetic. In many cases of theoretical approach due to complexity of the analytical calculation, it is necessary to do some simplification in a formulation of the problem. In Refs. 10 and 11, it was not taken into account that the gradient of electric field can essentially influence on the picture of mechanical oscillation in the systems with sharp interphase boundary. Also, the authors considered only one case of magnetostrictive oscillations along the plane of structure.
This work is devoted to the theoretical research of direct ME effect in bilayers of ferromagnetic and piezoelectric taking into account the nonuniform electric field along the layer thickness.

\section{Natural Frequences of the Bilayer System}

We have studied that the structure lies in the plane perpendicular to the $z$-axis (Fig. 1) and magnetic field $H_{z}=$ $H_{0} \cos \omega t$ with magnitude $H_{0}$ and oscillation frequency $\omega$ applies along $z$-axis, the vectors of polarization $\mathbf{P}$ of piezoelectric plane (2) and magnetization $\mathbf{M}$ of ferromagnetic plane (1). In this case, the phenomenon of longitudinal ME effect has occurred. The elastic oscillations appear in layer (1) under the influence of magnetic field $H_{z}$, which by means of shear deformations due to mechanical connection between layers are transmitted by layer (2), and as a result of piezoelectric effect, the elastic oscillation produces the variable electric field (Fig. 1).

We confine ourselves to consideration of a wide plate when the displacement of material along the $y$-axis can be presumed uniform. The displacement vector $\mathbf{u}^{(j)}\left(u_{x}, u_{z}\right), j=$ 1,2 and the electric potential $\varphi$ of the piezoelectric layer should be nonuniform across the sample thickness due to the sharply defined boundary between the materials.

\subsection{Uniform oscillations through the thickness of layers}

Consider the case of uniform oscillations through the thickness of layers. Oscillatory processes in the system described

This paper is submitted to the 2nd Russia-China Workshop on Dielectric and Ferroelectric Materials, Voronezh, Russia, Sept. 11-14, 2015.

This is an Open Access article published by World Scientific Publishing Company. It is distributed under the terms of the Creative Commons Attribution 4.0 (CC-BY) License. Further distribution of this work is permitted, provided the original work is properly cited. 


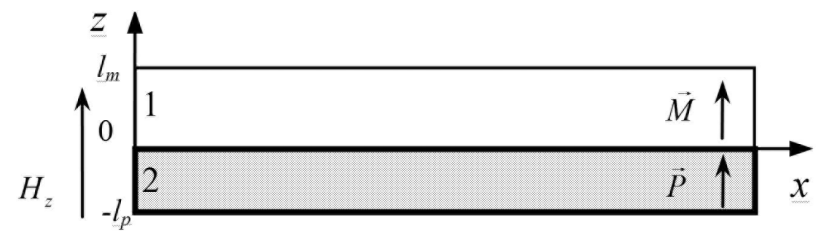

Fig. 1. The geometry of bilayer structure.

by the equations of motion of the contacting medium and Maxwell's equation in the absence of the external charges for the piezoelectric layer:

$$
\begin{gathered}
\rho^{(1)} \frac{\partial^{2} u_{x}^{(1)}}{\partial t^{2}}=\frac{\partial \sigma_{x x}^{(1)}}{\partial x}+\frac{\partial \sigma_{x z}^{(1)}}{\partial z} \\
\left\{\begin{array}{l}
\rho^{(2)} \frac{\partial^{2} u_{x}^{(2)}}{\partial t^{2}}=\frac{\partial \sigma_{x x}^{(2)}}{\partial x}+\frac{\partial \sigma_{x z}^{(2)}}{\partial z} \\
\operatorname{div} \mathbf{D}=0
\end{array}\right.
\end{gathered}
$$

where $\sigma_{x x}^{(1)}, \sigma_{x z}^{(1)}, \sigma_{x x}^{(2)}, \sigma_{x z}^{(2)}$ are the tensor components of mechanical stress in the layers, $\rho^{(1)}, \rho^{(2)}$ are the densities of ferromagnetic and piezoelectric materials, $\mathbf{D}\left(D_{x}, D_{z}\right)$ is the vector of electric displacement of piezoelectric layer. Taking into consideration the oscillation homogeneity in the layer plane and the Hooke's law, Eq. (1) for ferromagnetic layer and the system of equations (2) for piezoelectric layer have the form,

$$
\begin{gathered}
\rho^{(1)} \frac{\partial^{2} u_{x}^{(1)}}{\partial t^{2}}=\frac{G^{(1)}}{2} \frac{\partial^{2} u_{x}^{(1)}}{\partial z^{2}} \\
\left\{\begin{array}{l}
\rho^{(2)} \frac{\partial^{2} u_{x}^{(2)}}{\partial t^{2}}=\frac{G^{(2)}}{2} \frac{\partial^{2} u_{x}^{(2)}}{\partial z^{2}}-G^{(2)} d_{x z z} \frac{\partial E_{z}}{\partial z} \\
\frac{\partial D_{z}}{\partial z}=0
\end{array}\right.
\end{gathered}
$$

where $G^{(1)}, G^{(2)}$ are the shear modulus of materials, $d_{x z z}$ is the piezoelectric module, $E_{z}$ is the projection of electric field vector on the $z$-axis, determined through electric potential $\varphi$ of piezoelectric layer

$$
E_{z}=-\frac{\partial \varphi}{\partial z}
$$

where $D_{z}$ is the component of electric displacement vector

$$
D_{z}=-\varepsilon_{z z} \frac{\partial \varphi}{\partial z}+d_{x z z} \frac{G^{(2)}}{2} \frac{\partial u_{x}^{(2)}}{\partial z}
$$

where $\varepsilon_{z z}$ is the component of dielectric constant tensor along the $z$-axis.

Using the expressions (5) and (6), the system of equations (4) has the form,

$$
\left\{\begin{array}{l}
\rho^{(2)} \frac{\partial^{2} u_{x}^{(2)}}{\partial t^{2}}=\frac{G^{(2)}}{2} \frac{\partial^{2} u_{x}^{(2)}}{\partial z^{2}}+G^{(2)} d_{x z z} \frac{\partial^{2} \varphi}{\partial z^{2}} \\
\varepsilon_{z z} \frac{\partial^{2} \varphi}{\partial z^{2}}-d_{x z z} \frac{G^{(2)}}{2} \frac{\partial^{2} u_{x}^{(2)}}{\partial z^{2}}=0 .
\end{array}\right.
$$

The solutions of Eqs. (3) and (7) are convenient to find as

$$
\begin{aligned}
& u_{x}^{(j)}(t, z)=g^{(j)}(z) \mathrm{e}^{-i \omega t}, \quad j=1,2, \\
& \varphi(t, z)=h(z) \mathrm{e}^{-i \omega t} .
\end{aligned}
$$

The functions (8) are substituted in Eqs. (3) and (7) and the equations for the amplitudes of the required functions

$$
\begin{gathered}
-\rho^{(1)} \omega^{2} g^{(1)}=\frac{G^{(1)}}{2} \frac{\partial^{2} g^{(1)}}{\partial z^{2}}, \\
\left\{\begin{array}{l}
-\rho^{(2)} \omega^{2} g^{(2)}=\frac{G^{(2)}}{2} \frac{\partial^{2} g^{(2)}}{\partial z^{2}}+G^{(2)} d_{x z z} \frac{\partial^{2} h}{\partial z^{2}}, \\
\varepsilon_{z z} \frac{\partial^{2} h}{\partial z^{2}}-d_{x z z} \frac{G^{(2)}}{2} \frac{\partial^{2} g^{(2)}}{\partial z^{2}}=0 .
\end{array}\right.
\end{gathered}
$$

Taking into account that $C_{\perp}^{(j)}=\sqrt{G^{(j)} / \rho^{(j)}}$ are the speeds of transverse waves, and making the replacements

$$
C^{(1)}=\frac{C_{\perp}^{(1)}}{\sqrt{2}}, \quad C^{(2)}=\frac{C_{\perp}^{(2)}}{\sqrt{2}} \sqrt{1+\frac{d_{x z z}^{2}}{\varepsilon_{z z}} G^{(2)}},
$$

we rewrite Eqs. (9) and (10) in the form

$$
\frac{\partial^{2} g^{(j)}}{\partial z^{2}}+\left(\frac{\omega}{C^{(j)}}\right)^{2} g^{(j)}=0, \quad j=1,2,
$$

the general solutions of which have a form

$$
g^{(j)}(z)=A^{(j)} \mathrm{e}^{i \frac{\omega}{c^{(j)}} z}+B^{(j)} \mathrm{e}^{-i \frac{\omega}{c^{(j)}} z}, \quad j=1,2 .
$$

Using the conditions of smooth cross-linking functions $g^{(1)}$, $g^{(2)}$ and, considering the free plane structure, we obtain a homogeneous system of linear equations with coefficients $A^{(1)}, A^{(2)}, B^{(1)}, B^{(2)}$. The equality to zero of the determinant of this system gives a transcendental equation for determining the natural frequencies $\omega_{0}$ of structure oscillations:

$$
C_{1} \operatorname{tg}\left(\frac{\omega l_{p}}{C^{(2)}}\right)=-C_{2} \operatorname{tg}\left(\frac{\omega l_{m}}{C^{(1)}}\right) .
$$

The solution of this equation is found numerically (Fig. 2).

For numerically calculations, we use the parameters of permendur/PZT system: $M_{0}=480 \mathrm{Oe}, \quad C_{\perp}^{(1)}=3050 \mathrm{~m} / \mathrm{s}$, $\rho^{(1)}=8.2 \cdot 10^{3} \mathrm{~kg} / \mathrm{m}^{3}, \nu_{1}=0.34, E^{(1)}=205 \mathrm{GPa}, G^{(1)}=$ $76 \mathrm{GPa}, C_{\perp}^{(2)}=1830 \mathrm{~m} / \mathrm{s}, \rho^{(2)}=7.3 \cdot 10^{3} \mathrm{~kg} / \mathrm{m}^{3}, \nu_{2}=0.33$, $E^{(2)}=65 \mathrm{GPa}, \quad G^{(2)}=24 \mathrm{GPa}, \quad d_{x z z}=-175 \cdot 10^{-12} \mathrm{~m} / \mathrm{V}$, $\varepsilon_{z z} / \varepsilon_{0}=1750 .^{10,13}$

\subsection{Nonuniform oscillations through the thickness of layers}

Then, consider the case of nonuniform oscillations through the thickness of layers. Taking into account the nonuniform displacement across the layer thickness Eqs. (1) and (2) take the form,

$$
\left\{\begin{array}{l}
\rho^{(1)} \frac{\partial^{2} u_{x}^{(1)}}{\partial t^{2}}=\frac{\partial \sigma_{x x}^{(1)}}{\partial x}+\frac{\partial \sigma_{x z}^{(1)}}{\partial z} \\
\rho^{(1)} \frac{\partial^{2} u_{z}^{(1)}}{\partial t^{2}}=\frac{\partial \sigma_{z z}^{(1)}}{\partial z}+\frac{\partial \sigma_{x z}^{(1)}}{\partial x}
\end{array}\right.
$$




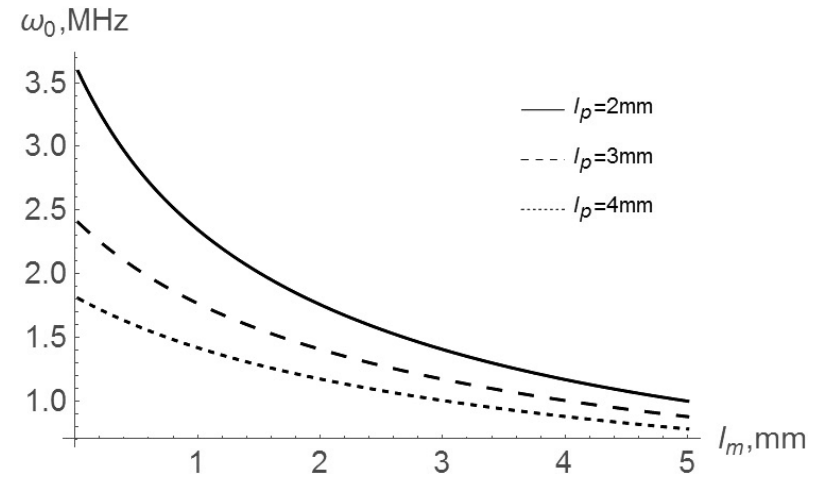

Fig. 2. The dependences of the natural frequency $\omega_{0}$ of the uniform oscillations through the thickness of structure on the thickness $l_{m}$ ferromagnetic layer for the different thickness $l_{p}$ of piezoelectric layer.

$$
\left\{\begin{array}{l}
\rho^{(2)} \frac{\partial^{2} u_{x}^{(2)}}{\partial t^{2}}=\frac{\partial \sigma_{x x}^{(2)}}{\partial x}+\frac{\partial \sigma_{x z}^{(2)}}{\partial z} \\
\rho^{(2)} \frac{\partial^{2} u_{z}^{(2)}}{\partial t^{2}}=\frac{\partial \sigma_{z z}^{(2)}}{\partial z}+\frac{\partial \sigma_{x z}^{(2)}}{\partial x} \\
\frac{\partial D_{x}}{\partial x}+\frac{\partial D_{z}}{\partial z}=0
\end{array}\right.
$$

As the previous case, when the stresses are expressed through displacements, we rewrite the systems of equations (11) and (12) in the form,

$$
\begin{gathered}
\left\{\begin{array}{c}
\rho^{(1)} \frac{\partial^{2} u_{x}^{(1)}}{\partial t^{2}}=E^{(1)} \frac{\partial^{2} u_{x}^{(1)}}{\partial x^{2}}+\frac{G^{(1)}}{2}\left(\frac{\partial^{2} u_{x}^{(1)}}{\partial z^{2}}+\frac{\partial^{2} u_{z}^{(1)}}{\partial x \partial z}\right) \\
\rho^{(1)} \frac{\partial^{2} u_{z}^{(1)}}{\partial t^{2}}=E^{(1)} \frac{\partial^{2} u_{z}^{(1)}}{\partial z^{2}}+\frac{G^{(1)}}{2}\left(\frac{\partial^{2} u_{z}^{(1)}}{\partial x^{2}}+\frac{\partial^{2} u_{x}^{(1)}}{\partial x \partial z}\right) \\
+\frac{G^{(2)}}{2}\left(\frac{\partial^{2} u_{x}^{(2)}}{\partial z^{2}}+\frac{\partial^{2} u_{z}^{(2)}}{\partial x \partial z}\right) \\
\rho^{(2)} \frac{\partial^{2} u_{x}^{(2)}}{\partial t^{2}}=E^{(2)}\left(\frac{\partial^{2} u_{x}^{(2)}}{\partial x^{2}}+d_{x z z} \frac{\partial^{2} \varphi}{\partial x \partial z}\right) \\
\rho^{(2)} \frac{\partial^{2} u_{z}^{(2)}}{\partial t^{2}}=E^{(2)} \frac{\partial^{2} u_{z}^{(2)}}{\partial z^{2}}+\frac{G^{(2)}}{2}\left(\frac{\partial^{2} u_{z}^{(2)}}{\partial x^{2}}+\frac{\partial^{2} u_{x}^{(2)}}{\partial x \partial z}\right) \\
\varepsilon_{\perp} \frac{\partial^{2} \varphi}{\partial x^{2}}+\varepsilon_{z z} \frac{\partial^{2} \varphi}{\partial z^{2}}-d_{x z z} \frac{G^{(2)}}{2}\left(\frac{\partial^{2} u_{x}^{(2)}}{\partial z^{2}}+\frac{\partial^{2} u_{z}^{(2)}}{\partial x \partial z}\right)=0
\end{array}\right.
\end{gathered}
$$

where $\varepsilon_{\perp}$ is the dielectric constant in the plane of structure. The solution of systems of equations (13) and (14) is convenient to find as

$$
\begin{array}{ll}
u_{x}^{(j)}(t, x, z)=g^{(j)}(z) \mathrm{e}^{i(k x-\omega t)}, & j=1,2, \\
u_{z}^{(j)}(t, x, z)=f^{(j)}(z) \mathrm{e}^{i(k x-\omega t)}, & j=1,2, \\
\varphi(t, x, z)=h(z) \mathrm{e}^{i(k x-\omega t)} .
\end{array}
$$

Firstly, we find the magnitudes $g^{(1)}(z), f^{(1)}(z)$ for this we substitute the functions (15) in the system of equations (13) and after simple manipulations, we obtain

$$
\left\{\begin{array}{l}
\frac{d^{2} g^{(1)}}{d z^{2}}+U^{(1)} g^{(1)}+i k \frac{d f^{(1)}}{d z}=0 \\
\frac{d^{2} f^{(1)}}{d z^{2}}+V^{(1)} f^{(1)}+\frac{i k}{4\left(1+\nu^{(1)}\right)} \frac{d g^{(1)}}{d z}=0
\end{array}\right.
$$

where

$$
\begin{gathered}
U^{(1)}=2\left(\frac{\omega}{C_{\perp}^{(1)}}\right)^{2}-4\left(1+\nu^{(1)}\right) k^{2}, \\
V^{(1)}=\left(2\left(\frac{\omega}{C_{\perp}^{(1)}}\right)^{2}-k^{2}\right) \frac{1}{4\left(1+\nu^{(1)}\right)} .
\end{gathered}
$$

The general solution of the system of equations (16) has the form

$$
\begin{aligned}
g^{(1)}(z)= & A_{1} \mathrm{e}^{\alpha_{1} z}+B_{1} \mathrm{e}^{\alpha_{2} z}+C_{1} \mathrm{e}^{-\alpha_{1} z}+D_{1}^{-\alpha_{2} z} \\
f^{(1)}(z)= & A_{1} M_{1} \mathrm{e}^{\alpha_{1} z}+B_{1} M_{2} \mathrm{e}^{\alpha_{2} z}+C_{1} M_{3} \mathrm{e}^{-\alpha_{1} z} \\
& +D_{1} M_{4} \mathrm{e}^{-\alpha_{2} z}
\end{aligned}
$$

where $A_{1}, B_{1}, C_{1}, D_{1}$ are the integration constants, $\alpha_{1,2}=\sqrt{R \pm \sqrt{R^{2}-U^{(1)} V^{(1)}}}$,

$$
\begin{gathered}
R=\frac{5+4 \nu^{(1)}}{4\left(1+\nu^{(1)}\right)}\left(\frac{\omega}{C_{\perp}^{(1)}}\right)^{2}-2\left(1+\nu^{(1)}\right) k^{2}, \\
M_{j}=\frac{\alpha_{j}}{i k V^{(1)}}\left(\alpha_{j}^{2}+U^{(1)}+\frac{k^{2}}{4\left(1+\nu^{(1)}\right)}\right), \quad j=1, \ldots, 4 .
\end{gathered}
$$

Analogically, we find the magnitudes $g^{(2)}(z), f^{(2)}(z), h(z)$, when substitute the functions (15) in the system of equations (14):

$$
\left\{\begin{array}{l}
\frac{d^{2} g^{(2)}}{d z^{2}}+U^{(2)} g^{(2)}+i k \frac{d f^{(2)}}{d z}+4\left(1+\nu^{(2)}\right) d_{x z z} i k \frac{d h}{d z}=0, \\
\frac{d^{2} f^{(2)}}{d z^{2}}+V^{(2)} f^{(2)}+\frac{i k}{4\left(1+\nu^{(1)}\right)} \frac{d g^{(2)}}{d z}=0, \\
\frac{d^{2} h}{d z^{2}}-\frac{\varepsilon_{\perp}}{\varepsilon_{z z}} k^{2} h-\frac{d_{x z z} G^{(2)}}{2 \varepsilon_{z z}}\left(\frac{d^{2} g^{(2)}}{d z^{2}}+i k \frac{d f^{(2)}}{d z}\right)=0,
\end{array}\right.
$$

where

$$
\begin{gathered}
U^{(2)}=2\left(\frac{\omega}{C_{\perp}^{(2)}}\right)^{2}-4\left(1+\nu^{(2)}\right) k^{2}, \\
V^{(2)}=\left(2\left(\frac{\omega}{C_{\perp}^{(2)}}\right)^{2}-k^{2}\right) \frac{1}{4\left(1+\nu^{(2)}\right)} .
\end{gathered}
$$


The general solution of the system of equations (18) has the form

$$
\begin{aligned}
f^{(2)}(z)= & A \mathrm{e}^{-i \lambda_{1} z}+B \mathrm{e}^{-i \lambda_{2} z}+C \mathrm{e}^{-i \lambda_{3} z}+D \mathrm{e}^{-i \lambda_{4} z} \\
& +F \mathrm{e}^{-i \lambda_{5} z}+G \mathrm{e}^{-i \lambda_{6} z} \\
g^{(2)}(z)= & A L_{1} \mathrm{e}^{-i \lambda_{1} z}+B L_{2} \mathrm{e}^{-i \lambda_{2} z}+C L_{3} \mathrm{e}^{-i \lambda_{3} z} \\
& +D L_{4} \mathrm{e}^{-i \lambda_{4} z}+F L_{5} \mathrm{e}^{-i \lambda_{5} z}+G L_{6} \mathrm{e}^{-i \lambda_{6} z} \\
h(z)= & A N_{1} \mathrm{e}^{-i \lambda_{1} z}+B N_{2} \mathrm{e}^{-i \lambda_{2} z}+C N_{3} \mathrm{e}^{-i \lambda_{3} z} \\
& +D N_{4} \mathrm{e}^{-i \lambda_{4} z}+F N_{5} \mathrm{e}^{-i \lambda_{5} z}+G N_{6} \mathrm{e}^{-i \lambda_{6} z}
\end{aligned}
$$

where $A, B, C, D, F, G$ are the integration constants. We introduced the designations:

$$
\begin{aligned}
L_{j}=\lambda_{j} & \left\{\lambda_{j}^{4}-\frac{2\left(1+\nu^{(2)}\right) d_{x z z}^{2} G^{(2)} k}{\varepsilon_{z z}} \lambda_{j}^{3}\right. \\
& -\left[U^{(2)}+V^{(2)}+\frac{k^{2}}{4\left(1+\nu^{(2)}\right)}-k^{2} \frac{\varepsilon_{\perp}}{\varepsilon_{z z}}\right] \lambda_{j}^{2} \\
& +\frac{2\left(1+\nu^{(2)}\right) d_{x z z}^{2} G^{(2)} k}{\varepsilon_{z z}}\left[V^{(2)}+\frac{k^{2}}{4\left(1+\nu^{(2)}\right)}\right] \lambda_{j} \\
& \left.+\left[U^{(2)}-k^{2} \frac{\varepsilon_{\perp}}{\varepsilon_{z z}}\right] V^{(2)}-\frac{k^{4}}{4\left(1+\nu^{(2)}\right)} \frac{\varepsilon_{\perp}}{\varepsilon_{z z}}\right\} \\
& \times \frac{4\left(1+\nu^{(2)}\right)}{k^{3} U^{(2)}} \frac{\varepsilon_{z z}}{\varepsilon_{\perp}}, \\
N_{j}= & -\frac{1}{d_{x z z} k^{4}} \frac{\varepsilon_{z z}}{\varepsilon_{\perp}}\left\{\lambda_{j}^{4}-\frac{2\left(1+\nu^{(2)}\right) d_{x z z}^{2} G^{(2)} k}{\varepsilon_{z z}} \lambda_{j}^{3}\right. \\
& -\left[U^{(2)}+V^{(2)}+\frac{k^{2}}{4\left(1+\nu^{(2)}\right)}\right] \lambda_{j}^{2} \\
& +\frac{2\left(1+\nu^{(2)}\right) d_{x z z}^{2} G^{(2)} k}{\varepsilon_{z z}}\left[V^{(2)}+\frac{k^{2}}{4\left(1+\nu^{(2)}\right)}\right] \\
& \left.\times \lambda_{j}+U^{(2)} V^{(2)}\right\}, \quad j=1,2, \ldots, 6 .
\end{aligned}
$$

Determination of the integration constants for the functions (17) and (19) requires the solution of a system of 10 linear equations. The first four equations are derived from the conditions of free planes of the structure, therefore the stresses on their $\sigma_{x z}^{(j)}=\sigma_{z z}^{(j)}=0, j=1,2$. Next, four equations follow from the condition of an ideal contact between layers when there are stresses $\sigma_{x z}^{(j)}=\sigma_{z z}^{(j)}, j=1,2$ and displacement vectors $\mathbf{u}^{(1)}=\mathbf{u}^{(2)}$ on the contact plane. The electric potential $\varphi=0$ on this plane due contact between dielectric and metal materials exist, the electric field strength $E_{z}=0$ at the lower boundary. The natural frequency $\omega_{0}$ of the structure is found from the condition of determinant equal to zero for the resulting homogeneous system of equations:

$$
\begin{aligned}
& {\left[i\left\{X^{\{6\}}-A^{\{6\}} X^{\{3\}}+Z^{\{6\}}\left(A^{\{4\}} X^{\{3\}}-X^{\{4\}}\right)\right\}\right.} \\
& \left.\quad+I_{32}\left(A^{\{6\}}-A^{\{4\}} Z^{\{6\}}\right)+I_{42} Z^{\{6\}}-I_{62}\right] \\
& \quad \times\left[i\left\{X^{\{5\}}-A^{\{5\}} X^{\{3\}}+Z^{\{5\}}\left(A^{\{4\}} X^{\{3\}}-X^{\{4\}}\right)\right\}\right. \\
& \left.\quad+I_{32}\left(A^{\{6\}}-A^{\{4\}} Z^{\{5\}}\right)+I_{42} Z^{\{5\}}-I_{52}\right]^{-1}
\end{aligned}
$$

$$
\begin{aligned}
& -\left[i\left\{Y^{\{6\}}-A^{\{6\}} Y^{\{3\}}+Z^{\{6\}}\left(A^{\{4\}} Y^{\{3\}}-Y^{\{4\}}\right)\right\}\right. \\
& \left.+J_{32}\left(A^{\{6\}}-A^{\{4\}} Z^{\{6\}}\right)+J_{42} Z^{\{6\}}-J_{62}\right] \\
& \times\left[i\left\{Y^{\{5\}}-A^{\{5\}} Y^{\{3\}}+Z^{\{5\}}\left(A^{\{4\}} Y^{\{3\}}-Y^{\{4\}}\right)\right\}\right. \\
& \left.+J_{32}\left(A^{\{5\}}-A^{\{4\}} Z^{\{5\}}\right)+J_{42} Z^{\{5\}}-J_{52}\right]^{-1}=0,
\end{aligned}
$$

where

$$
\begin{gathered}
X^{\{j\}=} \frac{S_{j 2} W_{32}-T_{32} R_{j 2}}{T_{42} W_{32}-T_{32} W_{42}} \frac{H_{42} W_{32}-H_{32} W_{42}}{W_{32}} \\
\quad+\frac{H_{32} R_{j 2}}{W_{32}}, \quad j=3,4,5,6, \\
Y^{\{j\}}=\frac{S_{j 2} W_{32}-T_{32} R_{j 2}}{T_{42} W_{32}-T_{32} W_{42}} \frac{K_{42} W_{32}-K_{32} W_{42}}{W_{32}} \\
\quad+\frac{K_{32} R_{j 2}}{W_{32}}, \quad j=3,4,5,6, \\
Z^{\{j\}}=\frac{A^{\{j\}}-B^{\{j\}}}{A^{\{4\}}-B^{\{4\}}, \quad j=5,6,} \\
A^{\{j\}=} \frac{U_{j}-}{U_{3}-} U_{2} P_{j} P_{3}, \quad B^{\{j\}}=\frac{V_{j}-V_{2} P_{j}}{V_{3}-V_{2} P_{3}}, \quad j=4,5,6,
\end{gathered}
$$

where

$$
\begin{aligned}
& H_{j 2}=H_{j}-H_{2} Q_{j}, \quad K_{j 2}=K_{j}-H_{2} Q_{j}, \\
& T_{j 2}=T_{j}-T_{2} Q_{j}, \quad W_{j 2}=W_{j}-W_{2} Q_{j}, \quad j=3,4, \\
& S_{j 2}=S_{j}-S_{2} P_{j}, \quad R_{j 2}=R_{j}-R_{2} P_{j}, \quad j=3,4,5,6 .
\end{aligned}
$$

We introduced the designations:

$$
\begin{aligned}
& I_{j}=\left(\lambda_{j} L_{j}-k\right)-\left(\lambda_{1} L_{1}-k\right) \frac{N_{j}}{N_{1}}, \quad J_{j}=\lambda_{j}-\lambda_{1} \frac{N_{j}}{N_{1}}, \\
& U_{j}=\left(\lambda_{j} L_{j}-k\right) \mathrm{e}^{-i \lambda_{j} l_{p}}-\left(\lambda_{1} L_{1}-k\right) \mathrm{e}^{-i \lambda_{1} l_{p}} \frac{N_{j}}{N_{1}}, \\
& S_{j}=1-\frac{N_{j}}{N_{1}}, \quad R_{j}=L_{j}-L_{1} \frac{N_{j}}{N_{1}}, \\
& P_{j}=\frac{\lambda_{j} \mathrm{e}^{-i \lambda_{j} l_{p}}-\lambda_{1} \mathrm{e}^{-i \lambda_{1} l_{p}}}{\lambda_{2} \mathrm{e}^{-i \lambda_{2} l_{p}}-\lambda_{1} \mathrm{e}^{-i \lambda_{1} l_{p}}}, \\
& T_{j}=M_{j}\left(1-\frac{\alpha_{j}}{\alpha_{1}} \mathrm{e}^{\left(\alpha_{j}-\alpha_{1}\right) l_{m}}\right), \\
& W_{j}=1-\frac{\alpha_{j} M_{j}}{\alpha_{1} M_{1}} \mathrm{e}^{\left(\alpha_{j}-\alpha_{1}\right) l_{m}}, \\
& V_{j}=\lambda_{j} \mathrm{e}^{-i \lambda_{j} l_{p}}-\lambda_{1} \mathrm{e}^{-i \lambda_{1} l_{p}} \frac{N_{j}}{N_{1}}, \\
& H_{j}=\left(\alpha_{j}+i k M_{j}\right)-\left(\alpha_{1}+i k M_{1}\right) \frac{\alpha_{j} M_{j}}{\alpha_{1} M_{1}} \mathrm{e}^{\left(\alpha_{j}-\alpha_{1}\right) l_{m}}, \\
& Q_{j}=\frac{\left(\alpha_{j}+i k M_{j}\right) \alpha_{1} M_{1}-\left(\alpha_{1}+i k M_{1}\right) \alpha_{j} M_{j}}{\left(\alpha_{2}+i k M_{2}\right) \alpha_{1} M_{1}-\left(\alpha_{1}+i k M_{1}\right) \alpha_{2} M_{2}}, \\
& j=3,4,5,6 \text {. }
\end{aligned}
$$

The solution of Eq. (20) was obtained numerically. Figure 3 presents the dependences of the natural frequency $\omega_{0}$ on the ferromagnetic layer thickness. 


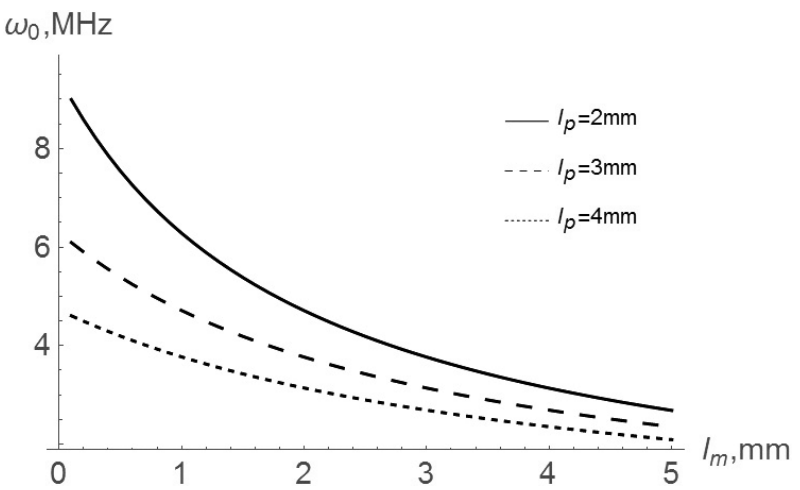

Fig. 3. The dependences of the natural frequency $\omega_{0}$ of the nonuniform oscillations through the thickness of structure on the thickness $l_{m}$ ferromagnetic layer for the different thickness $l_{p}$ of piezoelectric layer.

From Fig. 3, it follows that frequency of natural oscillation is reduced with increasing thickness of the magnetostrictive layer according to the law, which is close to the hyperbolic one. In the extreme case $(k \rightarrow 0)$, we obtain the exact relationship $\omega_{0}\left(l_{m}, l_{p}\right)$ :

$$
\omega_{0}=\frac{\pi}{\sqrt{2}} \frac{C_{\perp}^{(1)} C_{\perp}^{(2)}}{l_{m} C_{\perp}^{(1)}+l_{p} C_{\perp}^{(2)}} .
$$

Figure 3 shows that the natural frequency $\omega_{0}$ is significantly higher than one in the homogeneous case (Fig. 2). The difference in the curves increases with decreasing layers thickness that can be explained by the increased influence of nonuniform distribution of own electric fields in piezoelectric layer.

\section{Calculation of the ME Coefficient}

Based on the above results of the heterogeneous oscillations, we proceed to the calculation of the ME coefficient. Consider the case when the external mechanical stress $\sigma_{x x}$ is applied to ferromagnetic layer. Then the upper plane of composite should be satisfied to

$$
\sigma_{x z}^{(1)}=4 \pi M_{0} a_{2},
$$

where $a_{2}$ is the component of magnetostriction tensor. The nonuniform system of equations for determining the constants of integration are solved numerically. The constants found are substituted in solutions (17) and (19) and the equations on the magnitudes of the required functions are completely determined. To find the longitudinal ME voltage coefficient, we used the known relationship:

$$
\alpha_{E}=\frac{\left\langle E_{z}\right\rangle}{H_{0}},
$$

where $\left\langle E_{z}\right\rangle=\left.\varphi\right|_{z=l_{p}} / l_{p}$ is the electric field strength averaged over the thickness of the piezoelectric layer. The results of numerical calculation of the formula (21) are shown in Fig. 4.

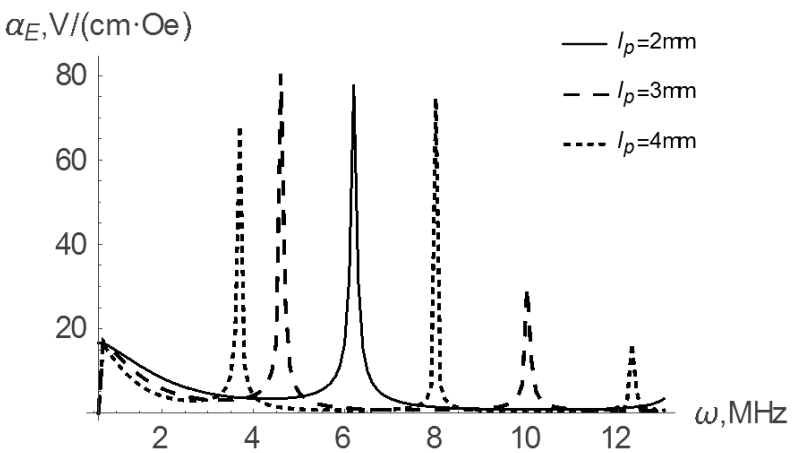

Fig. 4. The dependences of the longitudinal ME voltage coefficient $\alpha_{E}$ on the frequency $\omega$ of oscillation of external magnetic field $H_{z}$ at thickness of ferromagnetic layer $l_{m}=1 \mathrm{~mm}$.

Figure 4 shows that the resonance frequencies of ME effect correspond to the spectrum of natural frequencies of nonuniform oscillations of bilayer structure. The resonance frequencies are reduced when the piezoelectric layer thickness is increased, which is analogous to the behavior of natural frequencies $\omega_{0}$ (Fig. 3).

\section{Summary}

The dependences of natural mechanical oscillation on structure parameters numerically and analytically calculated in the cases of uniform and nonuniform oscillations through the thickness of layers. The frequency of natural oscillation is reduced with increasing thickness of the ferromagnetic layer according to the law, which is close to the hyperbolic one. The natural frequency of the nonuniform oscillations is significantly higher than one in the homogeneous case.

The dependence of longitudinal ME voltage coefficient on frequency of the variable magnetic field has been determined.

Values of the coefficient $\alpha_{E}$ for resonance frequencies are significantly larger than the values in the theoretical works. ${ }^{5,7,10}$ This fact, probably, can be explained by reduced depolarization field due to nonuniform distribution of electric potential on layer thickness.

\section{References}

${ }^{1}$ W. Eerenstein, N. D. Mathur and J. F. Scott, Multiferroic and magnetoelectric materials, Nature 442, 759 (2006).

${ }^{2}$ J. Ma, J. Hu, Z. Li and C. W. Nan, Recent progress in multiferroic magnetoelectric composites: From bulk to thin films, Adv. Mater. 23, 1062 (2011).

${ }^{3}$ M. Vopsaroiu, J. Blackburn and M. G. Cain, A new magnetic recording read head technology based on the magneto-electric effect, J. Phys. D, Appl. Phys. 40, 5027 (2007).

${ }^{4}$ T. Kimura, T. Goto, H. Shintani, K. Ishizaka, T. Arima and Y. Tokura, Magnetic control of ferroelectric polarization, Lett. Nature 426, 55 (2003).

${ }^{5}$ A. A. Bush, K. E. Kamentsev, V. F. Meshcheryakov, Y. K. Fetisov, D. V. Chashin and L. Y. Fetisov, Low-frequency magnetoelectric 
effect in a Galfenol-PZT planar composite structure, Tech. Phys. 54, 1314 (2009).

${ }^{6}$ G. Srinivasan, Magnetoelectric composites, Annu. Rev. Mater. Res. 40, 153 (2010).

${ }^{7} \mathrm{M}$. Fiebig, Revival of the magnetoelectric effect, J. Phys. D, Appl. Phys. 38, R123 (2005).

${ }^{8}$ R. Jungho, P. Shashank, U. Kenji and K. Hyoun-Ee, Magnetoelectric effect in composites of magnetostrictive and piezoelectric materials, J. Electroceram. 8, 107 (2002).

${ }^{9}$ A. V. Kalgin, S. A. Gridnev and Z. H. Gribe, Specific features of the inverse magnetoelectric effect in two-layered $\mathrm{Tb}_{0.12} \mathrm{Dy}_{0.68}$ $\mathrm{PbZr}_{0.53} \mathrm{Ti}_{0.47} \mathrm{O}_{3}$ composites, Phys. Solid State 56(7), 1327 (2014).
${ }^{10}$ D. A. Fillipov, Theory of magnetoelectric effect in ferromagneticpiezoelectric heterostructures, Phys. Solid State 47(6), 1118 (2005).

${ }^{11}$ M. I. Bichurin, D. A. Filippov, V. M. Petrov, V. M. Laletsin, N. Paddubnaya and G. Srinivasan, Resonance magnetoelectric effects in layered magnetostrictive-piezoelectric composites, Phys. Rev. B 68, 132408 (2003).

${ }^{12}$ C.-G. Duan, S. S. Jaswal and E. Y. Tsymbal, Predicted magnetoelectric effect in $\mathrm{Fe} / \mathrm{BaTiO}_{3}$ multilayers: Ferroelectric control of magnetism, Phys. Rev. Lett. 97, 047201 (2006).

${ }^{13}$ D. A. Filippov, V. M. Laletin and T. O. Firsova, Nonlinear magnetoelectric effect in composite multiferroics, Phys. Solid State 56(5), 980 (2014). 